\title{
Membrane stains as an objective means to distinguish isolated inner and outer hair cells
}

\author{
Gary Zajic a ${ }^{\text {, Andrew Forge }}{ }^{\mathrm{b}}$ and Jochen Schacht ${ }^{\text {at }}$ \\ "Kresge Hearing Research Institute, Unitersity of Michigan, Ann Arbor, Michigan, USA and "Institute of Laryngology and Otology. \\ Unitersity College London, London, UK
}

(Received 2 July 1992; Revision received 3 October 1992: Accepted 12 October 1992)

\begin{abstract}
The use of isolated cochlear outer and inner hair cells has become widespread. While the morphological features of these two cell types in general are sufficiently different to allow discrimination, there are situations where confusion can arise. Small outer hait cells. particularly when they are swollen or distorted. can take on an appearance suggestive of inner hair cells. We describe here two fluorescent membrane stains, 3,3'-dihexyloxacarbocyanine iodide and rhodamine B hexyl ester, as an objective means to distinguish between cochlear hair cell types. Both stains mark the subsurface cisternae of outer hair cells thereby delineating the cell outline, and the interior of the cell shows discrete structure. On the other hand, in inner hair cells, the outline of the cell is not resolved while the interior is diffusely fluorescent. Since the two probes have different excitation and emission wavelengths (fluorescein- and rhodamine-like, respectively), this staining procedure can even be used in the presence of another fluorescent marker (for example, a calcium-indicating dye) by appropriate choice of the membrane stain.
\end{abstract}

Hair cells, inner, outer: Membrane stains;

\section{Introduction}

Since the refined isolation of outer hair cells from the guinea pig cochlea (Brownell et al., 1985; Zenner et al., 1985), the use of these cells has become widespread in physiological, pharmacological, biochemical and physical-chemical investigations of their properties and functions. More recently, inner hair cells as well as supporting cells of the organ of Corti were isolated and received attention for their own properties and in comparison to characteristics of outer hair cells (Gitter and Zenner, 1990; Kros and Crawford, 1990; Dulon et al., 1991; Yamashita et al., 1990; 1991). Inner and outer hair cells show distinctive morphological features usually sufficient to distinguish these two species in vitro (Dulon et al., 1991). The major morphological criteria for a classification are the cylindrical shape of outer hair cells versus the pear-shaped appearance of inner hair cells with the cuticular plate residing at an oblique angle to the cell body. Other differences, such as size and position of the nucleus and length of the stereocilia in relation to cell body, are more subtle but can also be observed under light microscopy. Based on these distinctions, morphological

Correspondence to: Dr. Jochen Schacht, Kresge Hearing Research Institutc, University of Michigan, 1301 East Ann Street, Ann Arbor, MI 48109-0506, USA. Fax: (313) 764-0014. criteria should generally suffice to identify hair cell types. However, outer hair cells may resemble inner hair cells when they are mechanically distorted of begin swelling at their basal end. Thus, discrimination based solely on morphology can be problematic (Yamashita et al., 1991).

The problem of an exact determination under ambiguous circumstances is aggravated by the fact that the length of the cell - sometimes used as an differentiating argument - is not a reliable indicator. In basal turns of the guinea pig cochlea, the size ranges of inner and outer hair cells may overlap. More apically isolated outer hair cells can be up to $70-80 \mu \mathrm{m}$ long and thus clearly distinguishable from inner hair cells, which rarely exceed 30 to $40 \mu \mathrm{m}$ anywhere in the cochlea (Zajic and Schacht, 1987; Dulon et al., 1991). However, even in apical turns, length is not an unequivocal criterion since the size of an outer hair cell also depends on its row of origin. Outer hair cells in the innermost row are significantly smaller than their neighbors (Thalmann et al., 1972). Small outer hair cells can thus be found throughout the cochlea. In fact, cells primarily identified as inner hair cells on the basis of size (Yamashita et al., 1990) could more realistically be classified as outer hair cells by other morphological criteria (Dulon et al., 1991).

An objective means is necded to distinguish these two cell types unambiguously. Recently, we showed that outer hair cells exhibit a distinctive pattern of intracellular membrane staining with the fluorescent 
membrane probe 3,3'-dihexyloxacarbocyanine iodide $\left(\mathrm{DiOC}_{6}\right)$ (Forge et al., in press). Here we expand these findings and demonstrate cell-permeating, fluorescent membrane labels as practical tools to differentiate inner and outer hair cells.

\section{Materials and Methods}

\section{Cell preparation}

Cochleae were obtained from pigmented guinea pigs (150-200 g body weight), and cells were isolated from defined turns of the cochlea using procedures described by Dulon et al. (1990). Cells suspended in $50 \mu 1$ of Hanks' Balanced Salt Solution (HBSS) were allowed to settle and adhere to a glass coverslip placed over a hole cut into a Falcon $70 \mathrm{~mm}$ plastic petri dish.

\section{Fluorescence microscopy}

The membrane probes 3,3'-dihexyloxacarbocyanine iodide $\left(\mathrm{DiOC}_{6}\right)$ and rhodamine $\mathrm{B}$ hexyl ester chloride (R-6) were obtained from Molecular Probes (Eugene, $\mathrm{OR})$. Stock solutions of $\mathrm{DiOC}_{6}$ were prepared at 1 $\mathrm{mg} / \mathrm{ml}$ of ethanol and used on hair cells at a final concentration of $0.5 \mu \mathrm{g} / \mathrm{ml}$ HBSS. R- 6 was similarly dissolved to $1 \mathrm{mM}$ and used on hair cells at $0.5 \mu \mathrm{M}$. Dye was added to the droplet containing the hair cells for $1 \mathrm{~min}$ at room temperature and then diluted out by gently adding $10 \mathrm{ml}$ of HBSS to the coverslip. This brief treatment with the dye did not adversely affect hair cell morphology although the dyes can be cytotoxic (Terasaki and Reese, 1992). However, if the stain was left undiluted, degenerative changes in hair cells could be seen within 10 to $15 \mathrm{~min}$.

Cell preparations were observed with a MRC BioRAD 600 laser scanning confocal unit attached to a Nikon Diaphot TMD inverted microscope with a 60 $\times$ oil immersion objective, N.A. 1.40. The kryptonargon light source was usually attenuated with a 1.0 neutral density filter. Filter cubes for fluorescein con-

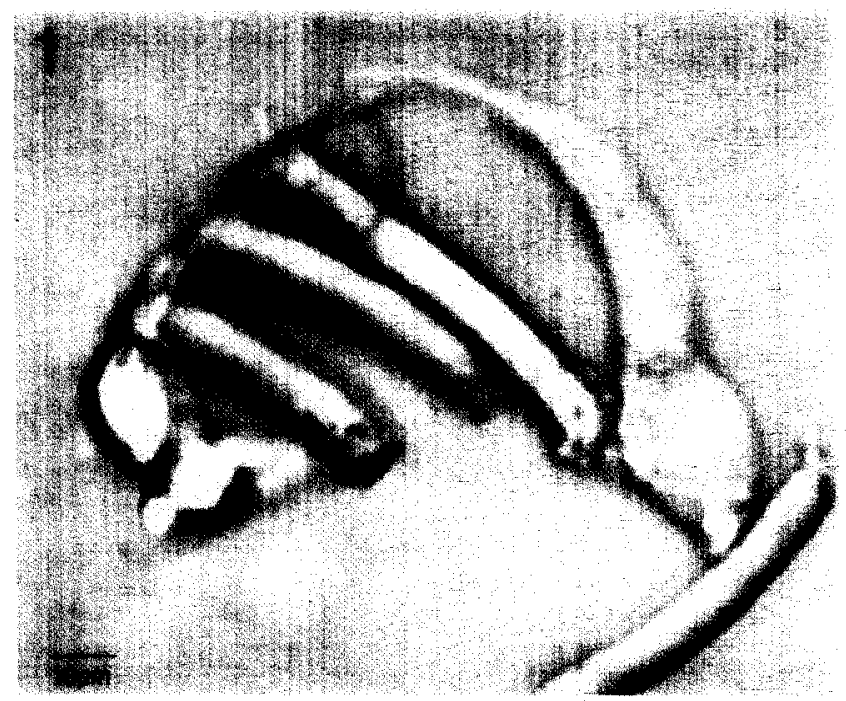

Fig. 1. Outer hair cells from rows 1 to 3 attached 10 a phalangeal process. Note the different length of cells from different rows.

tained an exciter filter at $488 \mathrm{~nm}$, dichroic reflector 510 LP (low pass) with a barrier or emission filter at 515 $\mathrm{nm}$ LP. The rhodamine cube contained a band pass filter at $514 \mathrm{~nm}$, a dichroic reflector at $540 \mathrm{~nm} \mathrm{LP}$ and emission filter at $550 \mathrm{~nm}$ LP. Fluorescent images were photographed from the video screen, bright field images through the microscope.

\section{Results}

Partially isolated outer hair cells still attached to the reticular lamina and a phalangeal process demonstrate the variation in size of cells of different rows (Fig. 1). In this specimen from an upper turn, the hair cells measure $68 \mu \mathrm{m}$ (outer row), $51 \mu \mathrm{m}$ (middle row) and $45 \mu \mathrm{m}$ (inner row).

Fig. 2a illustrates the relationship between outer and inner hair cells in-situ within a strip isolated from the middle turn of the cochlea. Outer hair cells are

Fig. 2. a) Partially isolated outer (left) and inner (right) hair cells from a lower turn. b and c). The same outer (2b) and inner hair cell (2c) as shown in Fig. 2a. Cells were fluorescently stained with DiOC $_{6}$ as described in 'Methods'.

Fig. 3. Outer hair cell from the basal region of the cochlea seen in bright field (3a) and fluorescently stained with DiOC 6 as described in 'Methods' (3b).

Fig. 4. Severely swollen outer hair cell from the same turn as cell in Fig. 3 seen in bright field (4a) and fluorescently stained with DiOC 6 as described in 'Methods' (4b).

Fig. 5. Apical outer hair cell stained with $\mathrm{DiOC}_{6}$ as described in 'Methods'

Fig. 6. Apical inner hair cell stained with $\mathrm{DiOC}_{6}$ as described in 'Methods'

Fig. 7. Apical outer hair cell stained with the membrane dye rhodamine B hexyl ester chloride as described in 'Methods'.

Fig. 8. Basal inner hair cell stained with the membrane dye rhodamine B hexyl ester as described in 'Methods' 
cylindrical in shape and the nucleus is located in the basal portion of the cell. Medial attachment of the outer hair cell to the head of the outer pillar cell is seen in the first row. The inner hair cell is pear-shaped and attached at its apical end to the head of the inner pillar cell. The apex of the cell is slanted so that the stereocilia are perpendicular to the reticular lamina. Upon isolation from the pillar cell, inner hair cells
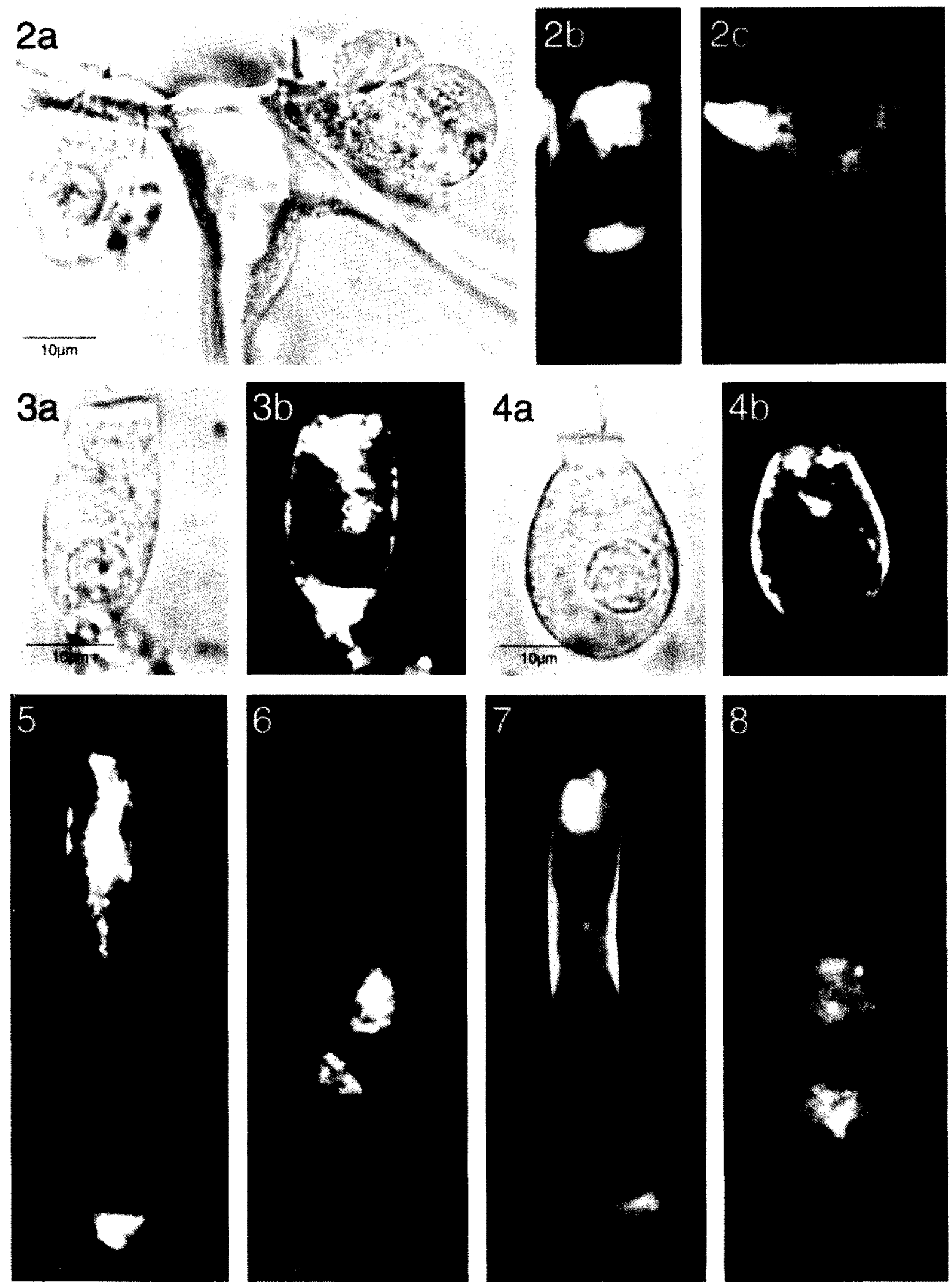
generally relax to a more flask-like shape. The nucleus is located higher in the central portion of the cell than in the outer hair cells. Typical of the majority of inner hair cells isolated, a swelling at the basal portion of the cell is evident. This region appears clear containing no intracellular organelles.

Staining with the intracellular membrane dye $\mathrm{DiOC}_{6}$ reveals discrete and different patterns in outer and inner hair cells (Fig $2 \mathrm{~b}$ and $2 \mathrm{c}$; Forge et al., in press). These patterns were consistent in all cells studied $(N>10$ for each cell type). The lipophilic carbocyanine dye is thought to stain the endoplasmic reticulum and also other membrane compartments including mitochondria, the Golgi apparatus, and lysosomes (Terasaki and Reese, 1992). Outer hair cells possess a complex system of subsurface cisternae along the lateral wall of the cell to nearly the level of the nucleus. Staining of this membrane system can easily be seen (Fig. 2b). Also seen are the membranes of numerous mitochondria located just below the nucleus as well as vesicles in the synaptic region. Staining is obvious along the periphery of the basal region of the cell and is intense in the region of the infracuticular network. This appears similar to the pattern seen with actin stains (Forge et al., in press). The cuticular plate and stereocilia remain free of fluorescence implying that the plasma membrane remains unstained.

In contrast, in the inner hair cell (Fig. 2c) staining is heavy in the infracuticular region and extends to both walls of the cell from below the cuticular plate to below the nucleus. However, the cell outline is barely detectable. Cell shape is defined more by the diffuse intracellular fluorescence than by a discrete membrane contour. In contrast to the basal portion of outer hair cells, the bottom portion of inner hair cells is not detected with this membrane dye. The contents of the swollen basal portion of the cell remains unstained.

Outer hair cells showing morphological signs of degeneration (Figs. $3 a$ and $4 a$ ) retain the distinctive staining of their outline (Fig. $3 \mathrm{~b}$ and $4 \mathrm{~b}$ ). Intracellular membranes are stained in the region of the infracuticular network while the cuticular plate and stereocilia are not seen. Mitochondria and synaptic vesicles are apparent in the basal region of the cell.

The staining patterns of inner and outer hair cells are consistent throughout the turns of the cochlea. This is evident in comparison of basal inner and outer hair cells (Fig. 2) to cells isolated from the apex (Figs. 5 and 6).

The membrane dye rhodamine $B$ hexyl ester provides an alternative to $\mathrm{DiOC}_{6}$ if fluorescence spectroscopy at a different wavelength is desired (Figs. 7 and 8). Staining with this dye results in essentially the same pattern as with DiOC $_{6}$ for both inner and outer hair cells.

\section{Discussion}

Either one of the fluorescent stains $\mathrm{DiOC}_{i}$ and $\mathrm{R}-6$ unquestionably discriminates inner and outer hair cells. Most strikingly, the cell contours are clearly outlined in outer hair cells while the outline of inner hair cells can only be inferred from the distribution of intracellular stain. Conversely, while the intracellular staining is quite diffuse in inner hair cells, it is distinct and limited to specific organelles in outer hair cells. The reason for this specific staining pattern is the fact that these probes for endoplasmic reticulum stain the subsurface cisternae along the lateral wall of outer hair cells, a structure absent from inner hair cells.

A salient point is that this staining pattern is consistent throughout the cochlea and remains unchanged when cells degenerate in culture. This is particularly important when outer hair cells lose their rigid cylindrical form due to the stress of isolation or incubation. Short outer hair cells, upon swelling and distortion, may then begin to resemble inner hair cells in shape. In fluorescence, however, these outer hair cells maintain their typical staining pattern with the plasma membrane clearly outlined. In fact, even completely deteriorated and rounded outer hair cells still stained in the specific endoplasmic reticulum pattern.

The dyes are potentially cytotoxic and should therefore be applied at the end of an experimental protocol. Since isolated hair cells are frequently investigated with fluorescent probes such as calcium indicators, it is important that the subsequent identification procedure is not compromised by the primary fluorescent probe. $\mathrm{DiOC}_{6}$ and R-6 fluoresce at different wavelengths, in the fluorescein and rhodamine region, respectively. Therefore, regardless of the original treatment of the hair cell, a complimentary staining procedure can be applied after the experiment in order to make an objective assessment of the hair cell in question.

\section{Acknowledgments}

This work was supported by NIH program project grant DC-00078 and a NATO Collaborative Research Award.

\section{References}

Brownell, W.E., Bader, C.R., Bertrand, D. and de Ribeaupierre, Y (1985) Evoked mechanical responses of isolated outer hair cells. Science 227, 194-196.

Dulon, D., Zajic, G. and Schacht, J. (1990) Increasing intracellular free calcium induces circumferential contractions in isolated outer hair cells. J. Neurosci. 10, 1388-1397.

Dulon, D., Zajic, G. and Schacht, J. (1991) Differential motile 
response of isolated inner and outer hair cells to stimulation by potassium and calcium. Hear. Res. 52, 225-231.

Forge, A.. Zajic. G., Li. L.. Nevill. G. and Schacht, J. (in press) Fluorescent labeling of intracellular membranes in viable outer hair cells. Hear. Res.

Gitter, A.11. and Zenner, H.P. (1990) The cell polential of isolated inner hair cells in vitro. Hear. Res. 45, 87-94.

Kros, C.J. and Crawford, A.C. (1990) Potassium currents in inner hair cells isolated from the guinea pig cochlea. J. Physiol. 421. 26.3-291.

Terasaki, M. and Reese, T.S. (1992) Characterisation of endoplasmic reticulum by colocalisation of $\mathrm{BiP}$ and dicarbocyanine dyes. $J$. Cell Sci. 101. 315-322.

Thalmann, R., Thalmann, I. and Comegys, T.H. (1972) Quantitative cytochemistry of the organ of Corti. Dissection, weight determination and analysis of single outer hair cells. Laryngoscope 82 , $2059-2078$.

Yamashita, T., Amano, H., Harada, N., Su. Z.L., Kumazawa, T., Tsunoda, Y. and Tashiro, Y. (1990) Calcium distribution and mobilization during depolarization in single cochlear hair cells. Acta Otolaryngol. 109, 256-262.

Yamashita, T., Amano, H., Ohtani, M., Harada, N. and Kumazawa, T. (1991) Isolation of and calcium kinetics in cochlear inner hair cells of the guinea pig. Acta Otolaryngol. 111. 879-84.

Zajic. G. and Schacht, J. (1987) Comparison of isolated outer hair cells from tive mammalian species. Hear. Res. 26, 249-256.

Zenner, H.P., Zimmermann. U. and Schmitt, U. (1985) Reversible contraction of isolated cochlear hair cells. Hear. Res. 18, 127-133. 